\title{
Experimental study of supercontinuum generation in step-index highly nonlinear fibers
}

\author{
Weiqing Gao, Lei Zhang, Haiwen Chen, Jingya Li, Yuxi Jiang, Lipeng Wan, Wenwen Dai, Wu Chen, Liang \\ Tong, Shaoqing Liu, Yong Zhou, and Xiaohui Ma ${ }^{1}$, \\ Department of Optical Engineering, School of Electronic Science \& Applied Physics, Hefei University of \\ Technology, Feicui Road 420, Hefei 230601, China \\ *gaoweiqing@hfut.edu.cn
}

\begin{abstract}
In this work, supercontinuum (SC) generation in three kinds of step-index highly nonlinear fibers (HNLFs) are experimentally investigated. The generated SC spectra are compared with each other by changing the numbers and wavelengths of the pump sources, and also by employing the HNLFs with different refractive-index differences between the core and the cladding. The widest spectral range of generated SC was about $2650 \mathrm{~nm}$ covering from 350 to $3000 \mathrm{~nm}$ when using the three pump wavelengths of 800,1400 and $1867 \mathrm{~nm}$. The results demonstrate that the stepindex HNLFs have excellent performances for SC generation and great potentials for the realization of all-fiber SC source.
\end{abstract}

\section{Keywords}

Supercontinuum; Step-index highly nonlinear fiber; all-fiber; 350-3000 nm;

\section{Introduction}

Since supercontinuum (SC) generation in optical fibers was first observed in 1976, it has been broadly applied in the applications on chemical sensing, optical coherence tomography, optical communication and microscopy [1-5]. However, the SC spectral range was restricted by the low nonlinear parameter and uncontrollable dispersion profile of the conventional fibers. It is well known that SC generation depends on the group-velocity dispersion (GVD), and typically involves the interplay of modulation instability (MI), self-phase modulation (SPM), four-wave mixing (FWM), Raman effect and soliton effects [6-8]. Therefore, proper selection of the fibers and the pump wavelengths is significant to generate the required SCs.

Photonic crystal fibers (PCFs) have been widely used in SC generation due to their adjustable GVD profiles and zero-dispersion wavelengths (ZDWs) [9-11]. It is usually structured by a strand of fused silica containing an array of microscopic hollow channels around a central glass core $[12,13]$. The SC spectrum can even be extended to midinfrared (MIR) region using PCF based on lead silicate glass, bismuth oxide glass, tellurite glass, fluoride glass, and chalcogenide glass [14-18]. However, the fusion between PCFs and standard single-mode fiber (SSMF) is quite difficult due to their different transverse structure and melting temperature $[19,20]$. When the pump lasers are coupled into the PCFs through a lens, great energy loss will be inevitable and the SC source is easy to be affected by the maladjustment. The highly nonlinear fibers (HNLFs) based on germanium-doped silica fiber may provide us a new thought. The nonlinear parameters of silica HNLFs are significantly larger than that of SSMF due to their small effective area [21-24]. In addition, silica HNLFs can be fusion spliced to SSMF directly, which provides the possibility for the SC source with all-fiber structure [25-28].

The pump source is also crucial to the SC generation. When a single pump wavelength is used, usually the spectral range and flatness of the generated SC are both limited. When two or more pump wavelengths are used, the spectral range and flatness can be greatly expanded and improved. Therefore, properly selecting the wavelengths and number 
of the pump sources could improve the SC generation with wider spectral range and higher spectral flatness.

In this work, the SC generations in step-index high nonlinear silica fibers are studied. The characteristics of SCs have been compared when one, two and three pump sources are used. In each case, the influence of the pump wavelength is also investigated. The SC generations in three kinds of HNLFs with different core and cladding refractive-index differences are also studied. These experimental results demonstrate that the step-index HNLFs have great performances in SC generation and great potentials for all-fiber SC sources.

\section{SC generation}

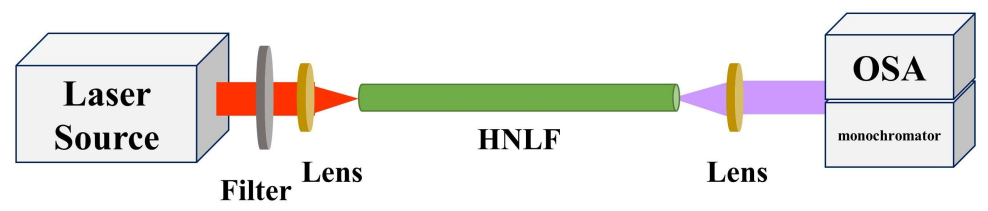

Fig. 1. The optical setup of SC generation.

The setup of SC generation is shown as in Fig. 1. The laser source was generated by an optical parametric oscillator (OPO) pumped by a Ti: sapphire laser with a wavelength of $800 \mathrm{~nm}$. The repetition rate was $76 \mathrm{MHz}$ and the full width at half maximum (FWHM) was $\sim 100 \mathrm{fs}$. The idler of the OPO could be tuned from 1.7 to $3.2 \mu \mathrm{m}$. The output spectra are measured by the optical spectrum analyzer (OSA) with a measurement range of 350-1200 nm (Yokogawa AQ6373) or 1200-2400 nm (Yokogawa AQ6375) and a monochromator. Then, the spectra measured by the three devices were connected together.

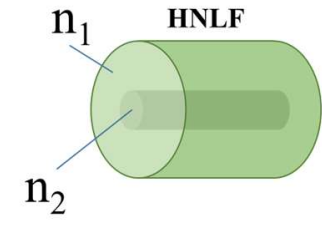

(a)

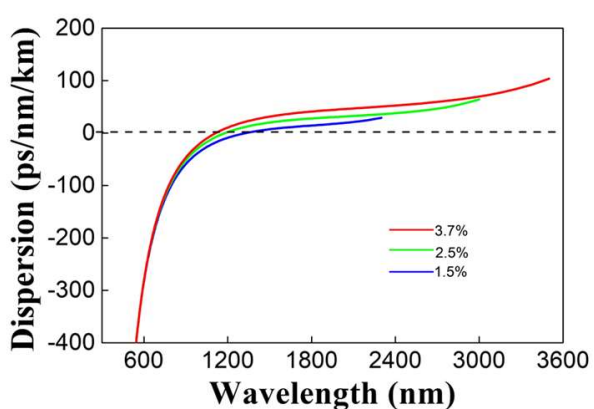

(b)

Fig. 2. (a) Scheme of cross section of the HNLF. (b) The dispersion profiles of the three HNLFs with different core and cladding refractive-index differences.

As shown in Fig. 2(a), the refractive indices of the cladding and the core of the HNLF are $n_{1}$ and $n_{2}$, respectively. The refractive index difference $\Delta$ is defined as $\Delta=\left(n_{2}-n_{1}\right) / n_{2}$, where $n_{2}$ is decided by the $\mathrm{GeO}_{2}$ concentration in the fiber core. The higher the doped concentration is, the higher the core refractive index is. In this work, three kinds of HNLFs with $\Delta$ of $3.7 \%, 2.5 \%$, and $1.5 \%$ are investigated for SC generation. The fiber core has a diameter of $4.4 \mu \mathrm{m}$. The dispersion profiles of the three HNLFs are shown as in Fig. 2(b), where the ZDWs are 1114, 1183, and $1342 \mathrm{~nm}$, respectively, decreasing with $\Delta$ increasing.

\subsection{The influence of pump source on SC generation}




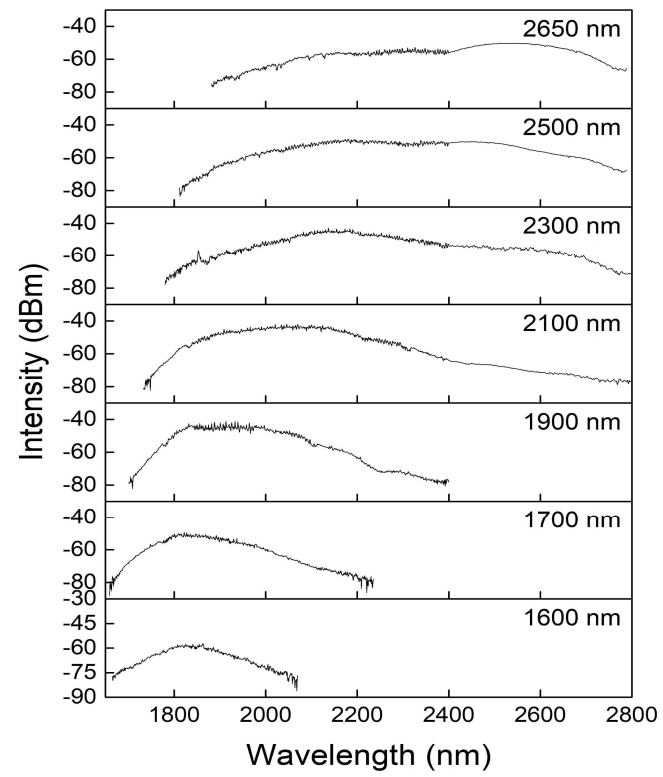

Fig. 3. The SC generation in the $1 \mathrm{~cm}$ long HNLF with $\Delta=3.7 \%$, pumped by the idler wave of OPO.

We studied the SC generation in the $1 \mathrm{~cm}$ long HNLF with $\Delta=3.7 \%$ when one, two and three pump sources were used. Firstly, the HNLF was pumped by the idler wave of OPO with the power of $1 \mathrm{~W}$. The wavelength was tuned from 1600 to $2650 \mathrm{~nm}$. A filter was used to remove the signal wave and the residual power of the Ti: sapphire laser, as shown in Fig.1. The generated SC is shown as in Fig. 3. All the pump wavelengths were in the anomalous dispersion region of the HNLF and far away from the ZDW. The SC was initiated by SPM and Raman effects. The soliton effects played an important role to extend the SC, including soliton self-frequency shift (SSFS) and soliton fission. Then, the spectra were extended further to longer wavelengths under the combined action of solitons, Raman, and FWM effects. In the spectral range shorter than the pump wavelength, the spectrum broadening at first was caused by the SPM effect. Then, the dispersive wave with shorter wavelengths began to emerge when the phasematching condition with the solitons was satisfied. The SC spectral range versus pump wavelength is shown as in Table 1, where the widest SC spectrum was about $1067 \mathrm{~nm}$ covering from 1733 to $2800 \mathrm{~nm}$ when the $2100 \mathrm{~nm}$ pump source was used.

Table 1. SC generation when one pump source was used.

\begin{tabular}{|c|c|c|c|c|c|c|c|}
\hline Pump source (nm) & 1600 & 1700 & 1900 & 2100 & 2300 & 2500 & 2650 \\
\hline Spectral range (nm) & $1665 \sim 2070$ & $1659 \sim 2236$ & $1701 \sim 2400$ & $1733 \sim 2800$ & $1780 \sim 2800$ & $1812 \sim 2788$ & $1822 \sim 2788$ \\
\hline Spectral width (nm) & 405 & 577 & 699 & 1067 & 1020 & 976 & 966 \\
\hline
\end{tabular}

Then, we studied the SC generation pumped by the signal and idler waves of OPO with both a power of $1 \mathrm{~W}$. A filter was used to remove the residual power of the Ti: sapphire laser. As shown in Fig. 4, the wavelengths of signal waves increased from 1150 to $1600 \mathrm{~nm}$, and the wavelengths of idler waves decreased from 2629 to $1600 \mathrm{~nm}$. 


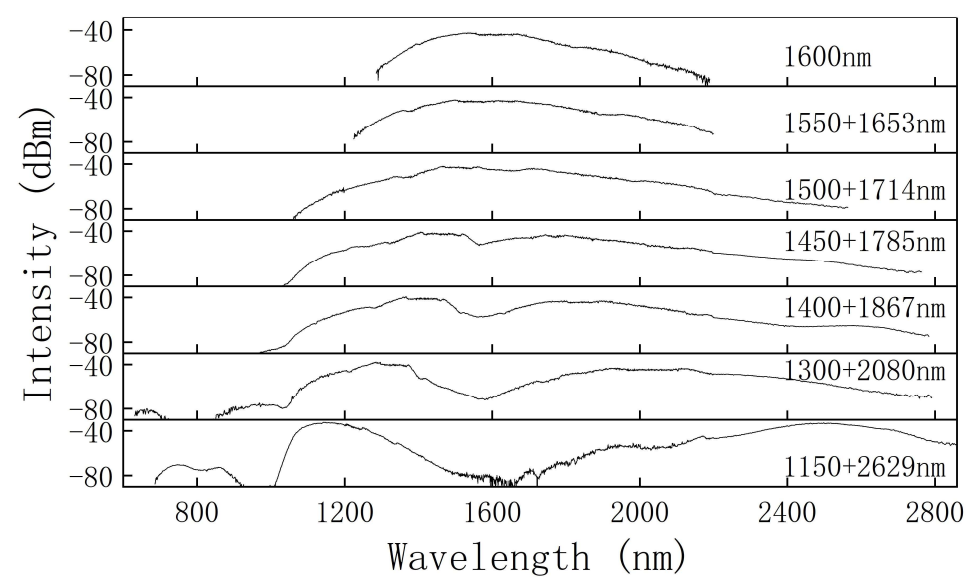

Fig. 4. The SC generation in the $1 \mathrm{~cm}$ long HNLF with $\Delta=3.7 \%$, pumped by the signal and idler wave of OPO.

For the pump wavelengths close to ZDW in the anomalous dispersion region of the HNLF, such as $1150 \mathrm{~nm}$ of the signal waves, the SC was initiated and extended to the longer wavelengths due to the soliton effects. The spectrum broadened to shorter wavelengths was caused by the SPM effect. When the broadened spectrum passed through the ZDW and entered the normal dispersion region, it was extended further by the SPM effect. At the same time, the interaction between solitons and pump waves results in the generation of dispersive waves, which generated new spectral components and continued to broaden the spectrum. It would also promote the FWM effect to move energy from the pulse center wavelength to the newly generated wavebands.

For the pump wavelengths far away from ZDW in the anomalous dispersion region of the HNLF, such as $1600 \mathrm{~nm}$, the broadened spectrum could not pass through the ZDW and enter the normal dispersion region. The SC generation was dominated by the interplay of MI, SSFS, SRS and FWM effects.

Table 2. SC generation when two pump sources were used.

\begin{tabular}{|c|c|c|c|c|c|c|c|}
\hline $\begin{array}{c}\text { Pump source } \\
\text { (signal + idler) } \\
(\mathbf{n m})\end{array}$ & $\begin{array}{c}1150+ \\
2629\end{array}$ & $\begin{array}{c}1300+ \\
2080\end{array}$ & $\begin{array}{c}1400+ \\
1867\end{array}$ & $\begin{array}{c}1450+ \\
1785\end{array}$ & $\begin{array}{c}1500+ \\
1714\end{array}$ & $\begin{array}{c}1550+ \\
1653\end{array}$ & $\begin{array}{c}1600+ \\
1600\end{array}$ \\
\hline Spectral range (nm) & $\begin{array}{c}686 \sim \\
2858\end{array}$ & $\begin{array}{c}631 \sim \\
2792\end{array}$ & $\begin{array}{c}970 \sim \\
2784\end{array}$ & $\begin{array}{c}1029 \sim \\
2764\end{array}$ & $\begin{array}{c}1061 \sim \\
2564\end{array}$ & $\begin{array}{c}1225 \sim \\
2200\end{array}$ & $\begin{array}{c}1286 \sim \\
2190\end{array}$ \\
\hline Spectral width (nm) & 2172 & 2161 & 1814 & 1703 & 1503 & 975 & 904 \\
\hline
\end{tabular}

The widest SC spectrum was about $2172 \mathrm{~nm}$ covering from 686 to $2858 \mathrm{~nm}$ when the signal and idler waves are $1150 \mathrm{~nm}$ and $2629 \mathrm{~nm}$. According to Fig. 4, when the wavelength difference between the signal and idler wave was too large, the flatness of the spectrum will decrease, especially in the middle part of the spectrum. Relatively flat spectrum could be got when the wavelength difference between the signal and idler wave was less than $200 \mathrm{~nm}$ (for example: $1500 \mathrm{~nm}$ (signal) and $1714 \mathrm{~nm}$ (idler)), although accompanied by a decrease in covering spectral range. 


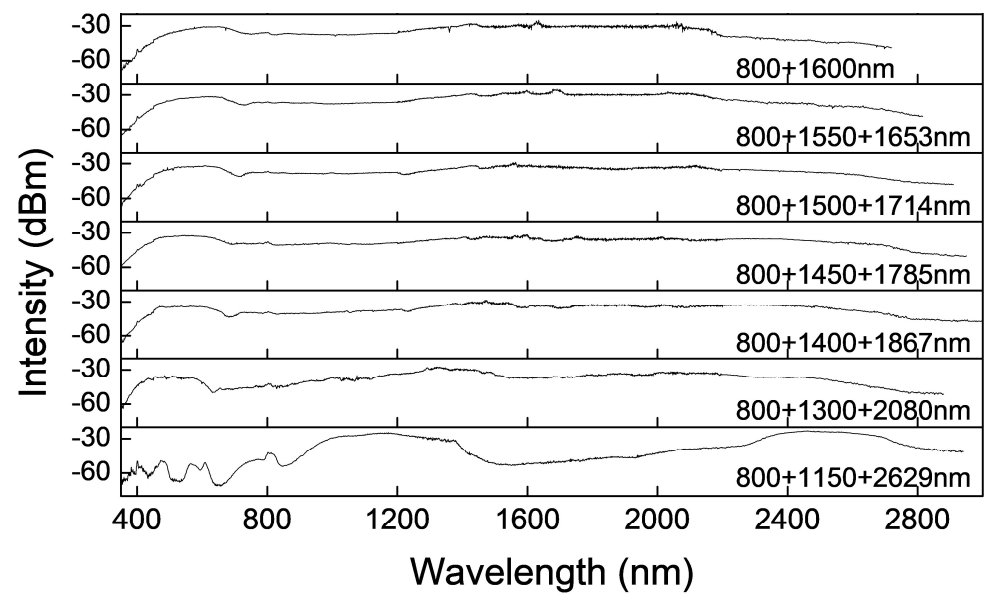

Fig. 5. The SC generation in the $1 \mathrm{~cm}$ long HNLF with $\Delta=3.7 \%$, pumped by the signal waves, idler waves and the residual pump source of the Ti: sapphire laser.

We also studied the SC generation pumped by three wavelengths, i.e., the signal and idler waves and the residual pump source of the Ti: sapphire laser. As shown in Fig. 5, the variation of the wavelengths of the idler and signal sources are exactly the same as those in Fig. 4.

For the pump wavelength of $800 \mathrm{~nm}$ in the normal dispersion region far from ZDW, the spectrum broadening was initialized by the SPM effect. The red-shifted components generated by the SPM effect transmitted faster than the blue-shifted components. As the optical pulses continued to propagate in the fiber, a large number of blue-shifted components generated by the SPM effect were retained at the trailing edge of the pulse, which makes the trailing edge of the pulse steeper, and the spectrum continues to broaden. After the spectra extended to longer wavelengths and reached the anomalous dispersion region, the Raman solitons were formed. Then the soliton peaks experienced broadening by the nonlinear effect as SPM and cross-phase modulation (XPM). In the spectral range shorter than 800 $\mathrm{nm}$, the spectrum broadening at first was caused by the SPM effect. Then, the dispersive wave ats shorter wavelengths began to emerge when the phase-matching condition with the Raman solitons was satisfied.

Table 3. SC generation when three pump sources were used.

\begin{tabular}{|c|c|c|c|c|c|c|c|}
\hline $\begin{array}{c}\text { Pump sources } \\
\text { (Ti: sapphire laser +signal + idler) } \\
(\mathbf{n m})\end{array}$ & $\begin{array}{c}800+ \\
1150+ \\
2629\end{array}$ & $\begin{array}{c}800+ \\
1300+ \\
2080\end{array}$ & $\begin{array}{c}800+ \\
1400+ \\
1867\end{array}$ & $\begin{array}{c}800+ \\
1450+ \\
1785\end{array}$ & $\begin{array}{c}800+ \\
1500+ \\
1714\end{array}$ & $\begin{array}{c}800+ \\
1550+ \\
1653\end{array}$ & $\begin{array}{r}800+ \\
1600+ \\
1600\end{array}$ \\
\hline Spectral range $(\mathrm{nm})$ & $\begin{array}{l}350 \sim \\
2944\end{array}$ & $\begin{array}{l}350 \sim \\
2882\end{array}$ & $\begin{array}{l}350 \sim \\
3000\end{array}$ & $\begin{array}{l}350 \sim \\
2950\end{array}$ & $\begin{array}{l}350 \sim \\
2916\end{array}$ & $\begin{array}{l}350 \sim \\
2816\end{array}$ & $\begin{array}{l}350 \sim \\
2724\end{array}$ \\
\hline Spectral width (nm) & 2594 & 2532 & 2650 & 2600 & 2566 & 2466 & 2374 \\
\hline
\end{tabular}

The widest SC was about $2650 \mathrm{~nm}$ covering from 350 to $3000 \mathrm{~nm}$ when the pump wavelengths are $800 \mathrm{~nm}$ (Ti: sapphire laser), $1400 \mathrm{~nm}$ (signal), and $1867 \mathrm{~nm}$ (idler). The spectral flatness was also much better than just using one or two pump sources.

\subsection{The influence of refractive index difference on SC generation}




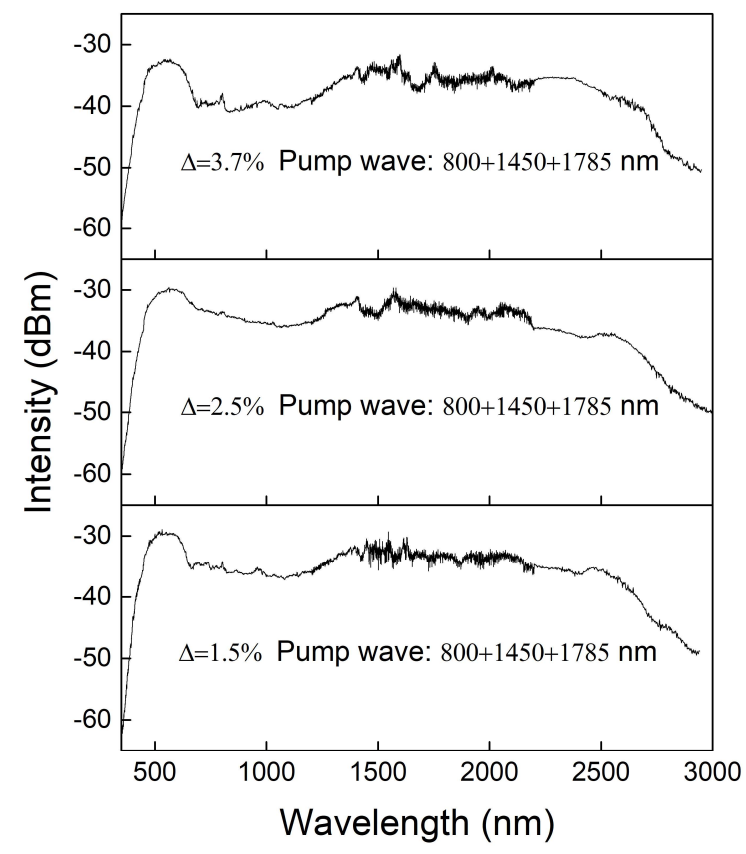

Fig. 6. SC generation in three HNLFs with different refractive index difference.

The influence of refractive index difference to SC generation was also studied. As shown in Fig. 6 , three $1 \mathrm{~cm}$ long HNLFs with $\Delta$ of $3.7 \%, 2.5 \%$ and $1.5 \%$ were studied when the pump wavelengths were $800 \mathrm{~nm}$ (Ti: sapphire laser), $1450 \mathrm{~nm}$ (signal), and $1785 \mathrm{~nm}$ (idler). The 10-dB bandwidth of the generated SC were 2297, 2223 and $2222 \mathrm{~nm}$, respectively. The results indicated that the refractive index difference has no significant influence on the spectral range and flatness of the generated SC when the pump power is high. The possible reasons are: 1) the pump peak power is high and generates very high nonlinear effect, then the nonlinear difference by the refractive index difference is covered by the effect of high peak power; 2) the fiber length is so short, so the effect on mode confinement by different refractive index difference is not obvious.

\section{Conclusions}

In this work, SC generation in different step-index HNLFs were studied when pumped by different wavelengths. The generated SCs by changing the number and wavelength of the pump sources were compared. When only one pump source was used, the widest spectral range of SC was about $1067 \mathrm{~nm}$ covering from $1733 \mathrm{~nm}$ to $2800 \mathrm{~nm}$ by employing the pump wavelength of $2100 \mathrm{~nm}$. When two pump sources were used, the widest SC spectral range was about $2172 \mathrm{~nm}$ covering from $686 \mathrm{~nm}$ to $2858 \mathrm{~nm}$ by employing the pump wavelengths of $1150 \mathrm{~nm}$ and $2629 \mathrm{~nm}$. Relatively flat spectrums could be got when the wavelength interval of the signal and idler was smaller than $200 \mathrm{~nm}$. When three pump sources were used, the widest spectral range of SC is about $2650 \mathrm{~nm}$ covering from $350 \mathrm{~nm}$ to $3000 \mathrm{~nm}$ by employing the pump wavelengths of $800 \mathrm{~nm}, 1400 \mathrm{~nm}$ and $1867 \mathrm{~nm}$. The spectral flatness was much better than just using one or two pump sources. We also investigated the generated SCs by changing the refractive index difference of HNLFs. When three HNLFs with refractive index differences of $3.7 \%, 2.5 \%$ and $1.5 \%$ were used, the 10-dB spectral range of the generated SC were $2297 \mathrm{~nm}, 2223 \mathrm{~nm}$ and $2222 \mathrm{~nm}$, respectively. The refractive index difference had no significant influence on the spectral range and flatness of the generated SCs. Our results demonstrate that the step-index HNLFs have good performances in SC generation and have great potential in realizing all-fiber SC source.

\section{Conflicts of interest}


The authors declare no conflicts of interest.

\section{Funding}

The work was supported by National Key R\&D Program of China (No. 2018YFB0504500). It was also supported by National Natural Science Foundation of China (NSFC) (Nos. 61875052 and 61905059), Anhui Provincial Natural Science Foundation (No. 1908085QF273), Fundamental Research Funds for the Central Universities (Nos. PA2019GDQT0007, JZ2020HGTB0065 and JZ2020HGQA0163), and Undergraduate Training Program for Innovation and Entrepreneurship (No. 202010359070).

\section{References}

1. C. Lin, and R. H. Stolen. "New nanosecond continuum for excited-state spectroscopy," Applied Physics Letters 28(4), 216-218 (1976).

2. C. F. Kaminski, R. S. Watt, A. D. Elder, J. H. Frank, and J. Hult, "Supercontinuum radiation for applications in chemical sensing and microscopy," Applied Physics B 92(3), 367 (2008).

3. S. Moon, and D. Y. Kim, "Ultra-high-speed optical coherence tomography with a stretched pulse supercontinuum source, " Optics Express 14(24), 11575-11584 (2006).

4. S. V. Smirnov, J. D. Ania-Castanon, T. J. Ellingham, S. M. Kobtsev, S. Kukarin, and S. K. Turitsyn, "Optical spectral broadening and supercontinuum generation in telecom applications," Optical Fiber Technology, 12(2), 122-147 (2006).

5. K. Lindfors, T. Kalkbrenner, P. Stoller, and V. Sandoghdar, "Detection and spectroscopy of gold nanoparticles using supercontinuum white light confocal microscopy," Physical Review Letters 93(3), 037401 (2004).

6. J. M. Dudley, and J. R. Taylor, "Supercontinuum generation in optical fibers, " Cambridge University Press (2010).

7. J. M. Dudley, G. Genty, and S. Coen, "Supercontinuum generation in photonic crystal fiber," Reviews of Modern Physics 78(4), 1135 (2006).

8. R. R. Alfano, "The Supercontinuum Laser Source: Fundamentals with Updated References," Springer Science \& Business Media (2006).

9. L. E. Hooper, P. J. Mosley, A. C. Muir, W. J. Wadsworth, and J. C. Knight, "Coherent supercontinuum generation in photonic crystal fiber with all-normal group velocity dispersion," Optics Express 19(6), 4902-4907 (2011)

10. W. Gao, M. El Amraoui, M. Liao, H. Kawashima, Z. Duan, D. Deng, T. Cheng, T. Suzuki, Y. Messaddeq, and Y. Ohishi, "Mid-infrared supercontinuum generation in a suspended-core $\mathrm{As}_{2} \mathrm{~S}_{3}$ chalcogenide microstructured optical fiber," Optics Express 21(8), 9573-9583 (2013).

11. A. Hartung, A. M. Heidt, and H. Bartelt, "Design of all-normal dispersion microstructured optical fibers for pulse-preserving supercontinuum generation," Optics Express 19(8), $7742-7749$ (2011).

12. R. Zhang, J. Teipel, and H. Giessen, "Theoretical design of a liquid-core photonic crystal fiber for supercontinuum generation," Optics Express 14(15), 6800-6812 (2006).

13. A. Ermolov, K. F. Mak, M. H. Frosz, J. C. Travers, and P. S. J. Russell, "Supercontinuum generation in the vacuum ultraviolet through dispersive-wave and soliton-plasma interaction in a noble-gas-filled hollow-core photonic crystal fiber," Physical Review A 92(3), 033821 (2015).

14. Z. Xing-Ping, L. Shu-Guang, D. Ying, H. Ying, Z. Wen-Qi, R. Yin-Lan, H. Ebendorff-Heidepriem, S. Afshar, and T. M. Monro, "High stability supercontinuum generation in lead silicate SF57 photonic crystal fibers," Chinese Physics B 22(1), 014215 (2013).

15. M. R. A. Moghaddam, S. W. Harun, R. Akbari, and H. Ahmad, "Flatly broadened supercontinuum generation in nonlinear fibers using a mode locked bismuth oxide based erbium doped fiber laser," Laser Physics Letters 8(5), 
369 (2011).

16. P. Domachuk, N. A. Wolchover, M. Cronin-Golomb, A. Wang, A. K. George, C. M. B. Cordeiro, J.C. Knight, and F. G. Omenetto, "Over $4000 \mathrm{~nm}$ bandwidth of mid-IR supercontinuum generation in sub-centimeter segments of highly nonlinear tellurite PCFs," Optics Express 16(10), 7161-7168 (2008).

17. G. Qin, X. Yan, C. Kito, M. Liao, C. Chaudhari, T. Suzuki, and Y. Ohishi, "Ultrabroadband supercontinuum generation from ultraviolet to $6.28 \mu \mathrm{m}$ in a fluoride fiber," Applied Physics Letters 95(16), 161103 (2009).

18. J. Hu, C. R. Menyuk, L. B. Shaw, J. S. Sanghera, and I. D. Aggarwal, "Maximizing the bandwidth of supercontinuum generation in $\mathrm{As}_{2} \mathrm{Se}_{3}$ chalcogenide fibers," Optics Express 18(7), 6722-6739 (2010).

19. Z. Zheng, D. Ouyang, J. Zhao, M. Liu, S. Ruan, P. Yan, and J. Wang, "Scaling all-fiber mid-infrared supercontinuum up to $10 \mathrm{~W}$-level based on thermal-spliced silica fiber and ZBLAN fiber," Photonics Research 4(4),135-139 (2016).

20. T. Hori, J. Takayanagi, N. Nishizawa, and T. Goto, "Flatly broadened, wideband and low noise supercontinuum generation in highly nonlinear hybrid fiber," Optics Express 12(2), 317-324 (2004).

21. N. Granzow, S. P. Stark, M. A. Schmidt, A. S. Tverjanovich, L. Wondraczek, and P. St. J. Russell, "Supercontinuum generation in chalcogenide-silica step-index fibers," Optics Express 19(21), 21003-21010 (2011).

22. C. R. Petersen, U. Møller, I. Kubat, B. Zhou, S. Dupont, J. Ramsay, T. Benson, S. Sujecki, N. Abdel-Moneim, Z. Tang, D. Furniss, A. Seddon, and O. Bang "Mid-infrared supercontinuum covering the 1.4-13.3 $\mu \mathrm{m}$ molecular fingerprint region using ultra-high NA chalcogenide step-index fibre," Nature Photonics 8(11), 830 (2014).

23. Y. Yu, B. Zhang, X. Gai, C. Zhai, S. Qi, W. Guo, Z. Yang, R. Wang, D. Choi, S. Madden, and B. Luther-Davies, "1.8-10 $\mu \mathrm{m}$ mid-infrared supercontinuum generated in a step-index chalcogenide fiber using low peak pump power," Optics Letters 40(6), 1081-1084 (2015).

24. T. Cheng, K. Nagasaka, T. H. Tuan, X. Xue, M. Matsumoto, H. Tezuka, T. Suzuki, and Y. Ohishi, "Mid-infrared supercontinuum generation spanning 2.0 to $15.1 \mu \mathrm{m}$ in a chalcogenide step-index fiber," Optics Letters 41(9), $2117-2120$ (2016)

25. W. Gao, M. Liao, L. Yang, X. Yan, T. Suzuki, and Y. Ohishi, "All-fiber broadband supercontinuum source with high efficiency in a step-index high nonlinear silica fiber," Applied Optics 51(8), 1071-1075 (2012).

26. W. Gao, M. Liao, X. Yan, T. Suzuki, and Y. Ohishi, "All-fiber quasi-continuous wave supercontinuum generation in single-mode high-nonlinear fiber pumped by submicrosecond pulse with low peak power," Applied Optics 51(13), 2346-2350 (2012)

27. J. W. Nicholson, M. F. Yan, P. Wisk, J. Fleming, F. DiMarcello, E. Monberg, A. Yablon, C. Jørgensen, and T. Veng, "All-fiber, octave-spanning supercontinuum," Optics Letters 28(8), 643-645 (2003).

28. R.R. Gattass, L. B. Shawa, V. Q. Nguyen, P. C. Pureza, I. D. Aggarwal, and J. S. Sangheraa, "All-fiber chalcogenide-based mid-infrared supercontinuum source," Optical Fiber Technology 18(5), 345-348 (2012). 